Revue d'histoire de l'Amérique française

THEYUE D.HISTOIRE DE L'AMÉRIQUE FRANÇAISE

ROCHER, Marie-Claude et Marc PELCHAT, dir., Le patrimoine des minorités religieuses du Québec. Richesse et vulnérabilité (Sainte-Foy, Les Presses de l’Université Laval, coll. « Patrimoine en mouvement », 2006), 273 p.

\title{
Lucia Ferretti
}

Volume 61, numéro 2, automne 2007

URI : https://id.erudit.org/iderudit/018076ar

DOI : https://doi.org/10.7202/018076ar

Aller au sommaire du numéro

Éditeur(s)

Institut d'histoire de l'Amérique française

ISSN

0035-2357 (imprimé)

1492-1383 (numérique)

Découvrir la revue

Citer ce compte rendu

Ferretti, L. (2007). Compte rendu de [ROCHER, Marie-Claude et Marc PELCHAT, dir., Le patrimoine des minorités religieuses du Québec. Richesse et vulnérabilité (Sainte-Foy, Les Presses de l'Université Laval, coll. « Patrimoine en mouvement ", 2006), 273 p.] Revue d'histoire de l'Amérique française, 61(2), 324-328. https://doi.org/10.7202/018076ar d'utilisation que vous pouvez consulter en ligne. 
géographiques (une seule, des traités historiques), alors qu'il aurait semblé judicieux de montrer, par exemple, la répartition des Autochtones dans le pays. On note également une certaine disparité entre les chapitres, qui est typique des collectifs, en particulier entre les notes biographiques, qui ne sont pas toutes de même qualité. Au-delà de ces aspects, le contenu et le projet global sont les bienvenus. Pour ma part, je conseillerai volontiers la lecture de cet ouvrage à mes étudiants pour les ressources pratiques qu'il fournit, comme ce tableau chronologique qui résume les relations entre les Autochtones et le gouvernement de 1945 à 2002 (p. 53-56), pour les voies d'investigation qu'il suggère et pour les sujets de réflexion qu'il génère. Outre aux étudiants, ce livre peut être recommandé à un public universitaire en général ainsi qu’à une plus large audience puisqu'il est facilement accessible. En fait, il ne faudrait justement pas le recommander uniquement à ceux qui se soucient des questions autochtones, mais aussi à ceux qui s'intéressent à l'art, à la littérature, à la justice, aux sports... Sinon, ne risquerait-on pas de passer à côté de son message?

MARIE-PIERRE BOUSQUET

Département d'anthropologie

Université de Montréal

ROCHER, Marie-Claude et Marc PELCHAT, dir., Le patrimoine des minorités religieuses du Québec. Richesse et vulnérabilité (Sainte-Foy, Les Presses de I'Université Laval, coll. «Patrimoine en mouvement», 2006), 273 p.

Cet ouvrage est issu d'un colloque organisé en 2006 dans le cadre du congrès annuel de l'ACFAS. À l'origine, la vingtaine de présentations étaient complétées de trois événements: une exposition d'éléments du patrimoine matériel de quelques minorités religieuses établies depuis longtemps au Québec; un accrochage au Musée McCord d'œuvres du peintre protestant d'origine suisse André Biéler, qui a passé une partie de sa jeunesse à Montréal et à l'Île d'Orléans avant de s'installer en Ontario; et un documentaire sur ce peintre réalisé par son petit-fils. La quatrième partie de l'ouvrage rend un pâle écho de tout cela (quoique en principe un DVD du film accompagne le livre, mais pas l'exemplaire expédié à la $R H A F)$. Cette partie contient aussi, en revanche, la remarquable allocution de clôture préparée par Daniel Turp (lui-même converti à la foi presbytérienne, si mon souvenir est bon) à titre de vice-président de la Commission sur la culture du Gouvernement du Québec: il a présenté les grandes 
lignes du rapport de celle-ci sur l'avenir du patrimoine religieux au Québec. Ceux qui se sont intéressés aux travaux de la Commission savent le sérieux et l'ouverture avec lesquels les commissaires ont reçu les personnes et groupes de tous horizons confessionnels et non confessionnels désireux de s'exprimer sur la question. Paru en 2006, Croire au patrimoine religieux du Québec est un document d'une qualité exceptionnelle, susceptible de donner confiance à tous ceux qui, au Québec (et on s'est aperçu lors des audiences publiques qu'ils sont très très nombreux), croient à l'importance de la préservation et de la mise en valeur du patrimoine religieux à la fois pour des raisons identitaires - religieuses et nationales - très affirmées, et pour d'autres qui relèvent de l'art et du tourisme. Il y a eu dans ces audiences et il y a dans ce Rapport l'expression d'un peuple qui se révèle profondément engagé envers son patrimoine religieux, dans sa diversité et son unicité, engagement dont on avait déjà pu prendre la mesure depuis une quinzaine d'années par les succès de la Fondation du patrimoine religieux du Québec. Il faut souhaiter que le Gouvernement du Québec s'engage fermement à suivre les recommandations de sa Commission de la culture et faire pression pour l'y pousser.

Comme dans tout colloque, les contributions ont été inégales. Dans la première partie de l'ouvrage sont rassemblées celles destinées à faire connaître l'histoire de quelques-unes des minorités religieuses du Québec. Certains des participants ont tendance à prétendre que la mémoire historique québécoise aurait "occulté» la contribution des minorités religieuses et présenté le Québec comme une société homogène francophone et catholique. Ce reproche, à mon avis, n'est pertinent que dans le cas des Canadiens français protestants. Pour le reste, même les historiens les plus nationalistes des périodes antérieures, tel Lionel Groulx parmi d'autres, n'ont jamais prétendu que le Québec était une société homogène francophone et catholique, ils ont toujours tenu compte de la présence des anglo-protestants et des juifs, qui ont eu depuis la fin du $\mathrm{xvIII}^{\mathrm{e}}$ siècle un rôle dominant dans l'activité économique de la métropole, du Québec et $\mathrm{du}$ Canada tout entier et une influence significative sur la vie politique. Mais il est un peu normal, pour des raisons d'identité, de langue et d'accès aux sources, que les membres des confessions religieuses minoritaires soient les premiers intéressés à rappeler la contribution proprement religieuse de leurs coreligionnaires à l'histoire du Québec. Pourtant, très peu l'ont fait jusqu'à présent. Cela est en train de changer, surtout pour ce qui concerne les protestants francophones, ce dont témoignent bien la table ronde autour de Chiniquy ainsi que les textes de Didier Poton, Richard 
Lougheed, Jeanne Djaballah, Jean-Louis Lalonde et Marie-Claude Rocher entre autres, tous intéressants. Parmi les contributions novatrices, celle de Stéphane Bigham sur les orthodoxes, complétée par celle de Paul Ladouceur dans la deuxième partie du colloque. Les orthodoxes forment au Québec plusieurs petites communautés divisées selon l'ethnie encore aujourd'hui, malgré les quarante ans d'efforts de l'Association du clergé orthodoxe du Québec pour les unir et les ancrer localement au-delà de leur sentiment premier d'appartenance aux Églises des vieux pays. Historiquement, les orthodoxes se sont implantés surtout à Montréal, en Abitibi et à Rawdon. Alors que dans le Nord-Ouest leur souvenir n'est aujourd'hui perpétué que par les citoyens désormais presque exclusivement québécois francophones d'origine catholique des villes de RouynNoranda et Val-d'Or, dont les autorités municipales ont pris la charge des lieux de culte désaffectés par le départ des orthodoxes, des communautés vivantes se maintiennent encore à Montréal et Rawdon; leur clergé espère que le certificat en études orthodoxes de l'Université de Sherbrooke qu'il a contribué à mettre sur pied avec les professeurs de cet établissement favorisera enfin leur adaptation locale et leur développement.

Les deuxième et troisième parties du livre sont à mon sens les plus intéressantes. On y voit la diversité des attitudes des confessions religieuses vis-à-vis de leur propre patrimoine matériel et immatériel et l'inégalité des ressources dont elles disposent pour le conserver et le mettre en valeur. La précarité de la situation des protestants francophones de vieille tradition est frappante. Ils semblent ne pouvoir absolument pas compter sur leurs coreligionnaires anglophones pour trouver les ressources financières propres à reconstituer et à revitaliser une histoire et un patrimoine qui furent, dans ce cas c'est exact, "occultés»; ni sur les nouvelles communautés protestantes évangéliques francophones, peu tournées vers l'histoire et qui ne se sentent pas les héritières des communautés francoprotestantes du passé. Heureusement, le Québec français dans son ensemble est prêt désormais à "réintégrer» l'expérience des Canadiens français protestants dans la mémoire commune et nombre de villages, où traditionnellement ont vécu les franco-protestants, remettent en valeur cet aspect de leur histoire et de leur patrimoine. Quant à elle, la communauté juive dispose au contraire de moyens considérables pour conserver sa mémoire et se doter d'institutions propres, grâce à l'implication et au vigoureux sens d'appartenance de ses membres et à l'appui du Gouvernement du Québec. Disposée à prêter des objets et des pièces d'archives aux institutions muséales communes, telles que le Musée des religions de 
Nicolet par exemple, elle encourage encore davantage la diffusion de son patrimoine par le biais de musées virtuels ou de centres d'exposition sous son contrôle.

Cela nous conduit naturellement à deux autres présentations fort intéressantes et instructives: celles de Jean-François Royal du Musée des religions, justement, et celle d'Yves Bergeron et Carole Charbonneau, qui ont étudié la représentation des minorités religieuses dans les expositions permanentes des musées nationaux au Québec. Il en ressort que les musées font leur possible pour présenter le patrimoine religieux des diverses communautés de foi présentes sur le territoire du Québec, mais qu'ils se heurtent tout de même à plusieurs obstacles. Le premier, c'est que ce ne sont pas toutes les traditions religieuses qui incarnent leur foi dans des objets vraiment significatifs; pour certaines, l'assemblée des croyants elle-même est le plus grand trésor du patrimoine. Par ailleurs, certaines traditions sont très faiblement représentées au Québec. Au recensement de 2001, sur 7,1 millions de Québécois, plus de 5,9 millions se sont déclarés catholiques : cela correspond tout de même à plus de $83 \%$ de la population; les sans-religion sont le deuxième groupe en importance; les protestants forment moins de $5 \%$ de la population québécoise; les musulmans, près de $2 \%$; les orthodoxes, $1,7 \%$; les juifs à peine plus de $1 \%$. Une représentation équitable laisse forcément beaucoup de place aux catholiques. Et enfin, il arrive que des communautés minoritaires ne consentent à prêter que si elles gardent le contrôle sur la présentation qui sera faite des objets, ce qui va un peu à l'encontre des règles acceptées dans le monde muséal. Malgré tout, des efforts réels sont consentis pour faire connaître à l'ensemble des Québécois la diversité des héritages et des confessions religieuses présents ici.

Les organisateurs ont déploré l'absence de communications sur l'héritage spirituel des Autochtones. Les protestants anglophones n'ont pas participé au colloque non plus, ni les musulmans. On peut le regretter. Ce dont on peut se réjouir par contre, c'est que les 3 participants sur 26 qui étaient des anglophones se sont adressés en français à l'auditoire. Un effet de l'ACFAS? Espérons mieux. En adoptant enfin le français dans toutes les manifestations de leur vie publique autres que leurs assemblées cultuelles, les membres des minorités religieuses manifesteront clairement leur désir d'intégration et d'enracinement dans la culture commune. Cela correspond tout à fait, comme le montre l'accueil qu'elle réserve désormais dans sa mémoire aux franco-protestants et le soutien financier qu'elle accorde au patrimoine des confessions minoritaires par l'action du Gouvernement 
du Québec et des municipalités concernées, au souhait de la majorité francophone d'héritage catholique. Le partage d'une mémoire commune de nos héritages confessionnels diversifiés ainsi que leur célébration passent par le partage pleinement consenti d'une langue publique commune, témoin d'un sentiment d'appartenance à un Québec commun. Merci à Marie-Claude Rocher et à Marc Pelchat pour ce colloque.

LUCIA FERRETTI

Département des sciences humaines Université du Québec à Trois-Rivières

RYAN, Pascale, Penser la nation. La Ligue d'Action nationale, 1917-1960 (Montréal, Leméac Éditeur, coll. «Domaine histoire», 2006), 324 p.

Penser la nation arrive à point nommé. Les études sur L'Action française et L'Action nationale, revue d'idées et de combats qui vient de célébrer son $90^{\mathrm{e}}$ anniversaire, ne manquent pas, mais aucune n'est pleinement satisfaisante. La plupart sont incomplètes, se concentrant sur un sujet précis ou une période restreinte. Ainsi, l'excellent mémoire de Jean-Claude Dupuis (1993) n'aborde que la période de L'Action française qui va de 1917 à 1928 (la revue renaîtra sous le nom d'Action nationale en 1933). L'essai récent de Catherine Bouchard, Les nations québécoises dans L'Action nationale (2002), se penche sur les années 1960-1990 et sur un seul thème - quoiqu'évidemment central. D'autres études sont devenues caduques, malgré leurs mérites, grâce aux progrès de la recherche; c'est le cas du livre de Susan Mann Trofimenkoff qui date du début des années 1970. D’autres enfin sont imprégnées d'idéologie «révolutionnaire-tranquille » à un point tel que leur téléologie les empêche d'admettre la possibilité d'une mouvance dynamique, désintéressée et cohérente avant 1960.

$\mathrm{Au}$ fil du temps et de l'évolution de L'Action française puis nationale, l'auteure présente les aspects changeants de la revue (la place intermittente dévolue aux livres et aux arts, entre autres) et ses constantes, son administration, son nombre d'abonnés, ses célèbres enquêtes, ses numéros spéciaux. On peut suivre un à un les membres de la Ligue par une chronique minutieuse des arrivées et des départs. De copieuses indications biographiques et bibliographiques sont fournies. Pascale Ryan nous convie à la rencontre d'un groupe composite de clercs, de professeurs et de membres des professions libérales de tous âges - L'Action nationale ne s'est jamais caractérisée par une cohésion générationnelle, contrairement 